\title{
BMJ Open Antidepressant use and interpersonal violence perpetration: a protocol for a systematic review and meta-analysis
}

\author{
Claire Keen (D) , ${ }^{1}$ James A Foulds, ${ }^{2}$ Melissa Willoughby (D) , ${ }^{1,3}$ \\ Giles Newton-Howes, ${ }^{4}$ Josh Knight (1) , ${ }^{5}$ Seena Fazel, ${ }^{6}$ Rohan Borschmann, ${ }^{1,3,7,8}$ \\ Stuart A Kinner, ${ }^{1,3,9,10,11}$ Jesse T Young (D) 1,3,12,13
}

To cite: Keen C, Foulds JA, Willoughby $\mathrm{M}$, et al. Antidepressant use and interpersonal violence perpetration: a protocol for a systematic review and meta-analysis. BMJ Open 2021;11:e043306. doi:10.1136/ bmjopen-2020-043306

- Prepublication history for this paper is available online. To view these files, please visit the journal online (http://dx.doi. org/10.1136/bmjopen-2020043306).

Received 30 July 2020 Revised 08 December 2020 Accepted 19 December 2020

Check for updates

(C) Author(s) (or their employer(s)) 2021. Re-use permitted under CC BY-NC. No commercial re-use. See rights and permissions. Published by BMJ.

For numbered affiliations see end of article.

Correspondence to

Ms Claire Keen;

claire.keen@unimelb.edu.au

\section{ABSTRACT}

Introduction There are conflicting perspectives as to whether antidepressant medication increases, decreases or has no effect on violence perpetration, impulsivity and aggressive behaviour. This is an important question given the widespread use of antidepressant medication and the significant medical, social, legal and health consequences of violence. We aim to: (1) systematically identify observational studies and randomised controlled trials that quantify the relationship between antidepressant use and interpersonal violence; (2) assess the quality of studies that quantify the relationship between antidepressant use and interpersonal violence and (3) estimate the pooled prevalence and measure of effect for the relationship between antidepressant use and interpersonal violence. Methods and analysis We will search MEDLINE, EMBASE, CINAHL, PsycINFO, PubMed and the Cochrane Library for relevant peer-reviewed literature. Our primary outcome is the perpetration of violent acts directed at others. Our secondary outcome is physical, interpersonal aggression measured through validated surveys. We will include randomised controlled trials, cohort studies and case-control studies that examine the association between the use of antidepressants and violence perpetration and/or physical aggression. No restrictions will be placed on the population. We will use the Methodological Standard for Epidemiological Research scale to assess the quality of included studies. We will provide an overview of the included studies and assess heterogeneity and publication bias. If there are sufficient studies, we will conduct meta-analyses to examine the possible association between antidepressants and violence, and undertake meta-regression to examine the effect of antidepressant class, length of follow-up, age of participants and population subgroups on the association between antidepressants and violence.

Ethics and dissemination No ethics approval is required. Our findings will be disseminated through a peer-reviewed journal article and conference presentations. PROSPERO registration details CRD42020175474.

\section{INTRODUCTION}

The use of antidepressants, particularly in high-income countries, has become commonplace. ${ }^{1-3}$ Between 2000 and 2017, consumption of antidepressants in Organisation for
Strengths and limitations

- The relationship between antidepressants and violence is an important topic with medical, social and legal implications in a conflicting literature that hitherto has not been systematically reviewed.

- This systematic review uses a comprehensive search strategy including six key academic databases and a grey literature search.

- The review will be reported according to Preferred Reporting Items for Systematic Reviews and MetaAnalysis guidelines for conducting and reporting systematic reviews.

- The planned analysis will allow for clinically relevant covariates to be examined, if sufficient evidence is found.

- A small number of primary studies, and heterogeneity between study populations and study design, may preclude meta-analysis or direct comparisons between studies.

Economic Co-operation and Development countries doubled. ${ }^{4}$ While this may, in part, reflect higher doses in long-term users of antidepressants due to increasing tolerance, it also likely reflects a growing proportion of the population using antidepressants. In Australia, the United States and England, it is estimated that more than 1 in 10 adults were prescribed an antidepressant in the most recent year that data are available. ${ }^{356}$ In addition to treating depression, antidepressants are also used for a range of other conditions, such as anxiety disorders, insomnia and pain. ${ }^{7}$

Interpersonal violence is a serious public health concern. ${ }^{8}$ In 2013, the Global Burden of Disease (GBD) study estimated there were approximately 405000 deaths globally due to interpersonal violence. ${ }^{9}$ In 2017 , the GBD estimated there were approximately 4.5 million years lived with disability due to interpersonal violence. ${ }^{10}$ Given the profound impacts of violence, societies have consistently attempted to control aggressive behaviour 
and prevent violence. ${ }^{8}$ Accordingly, the use of pharmacotherapy to modulate aggressive and violent behaviour has been examined for more than four decades. Neuropsychiatric agents including lithium, ${ }^{11}$ benzodiazepines, ${ }^{12}$ atypical antipsychotics ${ }^{13} 14$ and antiepileptic drugs ${ }^{15}$ have all been examined as potential pharmacotherapies for reducing violent and aggressive behaviour. To date, however, none of these pharmacotherapies have shown long-term effectiveness. ${ }^{16}$

Antidepressants, including selective serotonin reuptake inhibitors (SSRIs), have also been identified as potential candidates for violence-prevention medications. ${ }^{17} 18$ The use of serotonergic or noradrenergic antidepressants to reduce violent behaviour has some biological plausibility. Serotonergic dysfunction has been associated with increased aggressive behaviour in animal and human studies. ${ }^{19-21}$ Similarly, the noradrenergic system has been found to modulate aggressive behaviour in animal studies, and a hyperactive noradrenergic system has been correlated with aggressive behaviour in humans. ${ }^{22}$ Therefore, drugs that regulate the serotonergic or noradrenergic systems may act to reduce aggressive and violent behaviour. Indeed, some research has reported an association between the use of SSRIs and reduced impulsivity and aggressive behaviour. ${ }^{1823-26}$

However, contrasting research findings have also emerged, with some studies reporting an association between the use of antidepressants and increased aggression and violence perpetration. ${ }^{27-31}$ For example, a systematic review of clinical study reports found an increased risk of aggression in children and adolescents while using SSRIs but not in adults. ${ }^{27}$ A second systematic review of randomised control trials (RCTs) examining the association between antidepressants, suicidality and violence in healthy volunteers found that taking antidepressants doubled the occurrence of events, such as anxiety, nervousness and agitation, that may serve as proxy markers for violence. ${ }^{28}$ Similarly, an expert review argued that clinical trial and pharmacovigilance data suggest that SSRIs are associated with an increased violent behaviour. $^{30}$

Compounding the conflicting nature of this evidence, the methodology of this research, particularly the published systematic reviews, has been criticised. Concerns include: the use of adverse events and/or proxy measures of violence that are only tangentially related to violence, limitations of the statistical analysis techniques used and inaccuracies in the authors' interpretation of the included studies. ${ }^{32-36}$ Further, most systematic reviews on antidepressants and violence have had a primary focus on violence against self, with limited or no examination of violence against others. ${ }^{27} 2837$ These limitations have led some researchers to argue that the assertion that there is an association between antidepressant use and increased aggression or violence against others is overstated or misleading. ${ }^{35}$

Others have argued that weaknesses of the included RCTs may have resulted in an under-reporting of violence perpetration related to antidepressants. ${ }^{27}$ Measures of violent behaviour, and related adverse events such as aggression and impulsivity, have been poorly recorded in many RCTs. ${ }^{27} 2838$ Further, the majority of RCTs have little capacity to examine long-term harms such as violence due to short follow-up periods, ${ }^{38}$ small sample size and strict participant inclusion criteria that may exclude those at high risk of aggression or violence ${ }^{18}$ and the relative rarity of violent events. Therefore, there is a reliance on proxy measures for violence such as self-reported aggression or hostility. ${ }^{182839}$ As such, the effectiveness of antidepressants in reducing violent outcomes is uncertain. ${ }^{39}$

Given the contradicting associations found between studies, concerns raised about prior review methodology and a possibility for bias in individual studies, a comprehensive review of the evidence on the association between antidepressant use and interpersonal violence perpetration is required. This is made more urgent by emerging recommendations for antidepressants to be used as a treatment for violent behaviour. ${ }^{17}$ Despite this, to our knowledge, there has been no attempt to systematically and rigorously synthesise both RCTs and observational studies that examine the association between antidepressant use and interpersonal violence.

This study seeks to examine the following key questions: to what extent has the association between antidepressant use and violence against others been investigated, what is the quality of research examining this association and what is the nature of the association (if present) between antidepressant use and violence against others? Therefore, in this study we will: (1) systematically identify RCTs and observational studies that quantify the relationship between antidepressant use and interpersonal violence perpetration; (2) assess the quality of studies that quantify the relationship between antidepressant use and interpersonal violence perpetration and (3) estimate the pooled prevalence and measure of effect for the relationship between antidepressant use and interpersonal violence perpetration.

\section{METHODS AND ANALYSIS}

This protocol adheres to the Preferred Reporting Items for Systematic Reviews and Meta-analysis for Protocols ${ }^{40}$ and has been registered with PROSPERO.

\section{Eligibility criteria}

Inclusion and exclusion criteria are outlined in table 1.

\section{Participants}

There are no restrictions on the participants of the reviewed studies. We will include studies that focus on the use of antidepressants in children, adults or specific subpopulations such as people with autism spectrum disorder, people with dementia, people with conduct disorder and people with a history of violent crime.

\section{Exposure measures}

We will include studies of antidepressants licensed as such for use by the US Food and Drug Administration (FDA), 
Table 1 Summary of inclusion and exclusion criteria

\begin{tabular}{|c|c|c|}
\hline & Inclusion criteria & Exclusion criteria \\
\hline Participants & No restrictions & N/A \\
\hline Intervention & Antidepressants (see table 2) & $\begin{array}{l}\text { Other psychotropic medications, antidepressant use of } \\
\text { less than } 1 \text { week }\end{array}$ \\
\hline Comparator & $\begin{array}{l}\text { Placebo, active comparator or periods of non-use } \\
\text { (self-controlled) }\end{array}$ & No comparison group \\
\hline Outcome & $\begin{array}{l}\text { Primary: acts of physical violence against others } \\
\text { Secondary: acts of physical aggression against } \\
\text { others measured through validated measure }\end{array}$ & $\begin{array}{l}\text { Verbal aggression, violence against self, self-reported } \\
\text { violence where type of violence is not specified, self- } \\
\text { reported violence through a non-validated measure, } \\
\text { measures of anger and irritability }\end{array}$ \\
\hline Study design & $\begin{array}{l}\text { Case-control studies, cohort studies and } \\
\text { randomised controlled trials }\end{array}$ & $\begin{array}{l}\text { Open-label single-arm trials, case reports, case series, } \\
\text { uncontrolled before/after studies, ecological studies and } \\
\text { cross-sectional studies }\end{array}$ \\
\hline
\end{tabular}

the UK Medicines and Healthcare products Regulatory Agency, the European Union's European Medicines Agency and Australia's Pharmaceutical Benefits Advisory Committee (PBAC) as of January 2020. These regulatory agencies were chosen as they regulate medications in the regions where rates of antidepressant prescribing are highest and have clear procedures for assessing and regulating medications. ${ }^{4} \mathrm{~A}$ list of included antidepressants is presented in table 2 .

We will include studies that compare the risk of violence perpetration between: (1) people exposed to antidepressants and people not exposed to antidepressants, including people exposed to medications other than antidepressants; (2) people exposed to different antidepressants or (3) time periods of exposure to antidepressants versus periods of no exposure, within individual participants. For example, we will include RCTs that use a placebo, an active control of a different antidepressant or other treatment or a crossover design. As antidepressants exert their effect via homeostatic changes that occur with repeated administration, we will require antidepressants to be used for at least 1 week for the study to be included. We will apply the same comparator criteria to RCTs and observational studies. We will exclude studies that examine antidepressant discontinuation, primarily use medication adherence as the exposure and in which the antidepressant medication is used recreationally rather than as prescribed by a health practitioner.

\section{Outcome measures}

Our primary outcome will be the perpetration of physical violence (ie, the intentional use of force) directed at others. ${ }^{41} 42$ This will include violent crimes such as homicide, assault, family or intimate partner violence, robbery, sexual assaults and self-reported, familial-reported, friend-reported or clinician-reported violent acts. This may be measured by self-report, administrative data (eg, ambulance, emergency department, hospital or criminal justice system records) or a valid observer rating scale. Consistent with our definition of interpersonal violence, we will exclude outcomes related to self-harm or suicide.
Given the difficulties in measuring violent acts directly, ${ }^{41}$ we will include measures of physical aggression ascertained through validated tools as secondary outcomes. There are plausible neurobiological mechanisms through which certain antidepressants may impact the occurrence of violent acts by reducing aggression. ${ }^{19-21}$ While we recognise that violence and aggression are dimensional, our focus is on externalised violent and aggressive physical actions and behaviours towards others, rather than studies which examine emotions, thoughts or verbalised aggression exclusively. As such, we will distinguish between physically aggressive acts towards others and the emotions or feelings that may be associated with such acts, including anger and irritability. Measures of the former will be included, whereas measures of the latter will not. We will exclude studies that use an experimental paradigm of aggression, due to a lack of generalisability.

\section{Study design}

We will include case-control studies, cohort studies and RCTs defined according to the Cochrane study design guide. ${ }^{43}$ Reviewing and synthesising the observational research will address a critical gap in the evidence on the potential association between antidepressant use and violence perpetration. Compared with RCTs, observational research typically allows for longer follow-up time, can include violence as the main study outcome with fewer ethical concerns and can examine the outcomes of a broader participant group under naturalistic (ie, real-world) circumstances. Studies using within-case comparisons (eg, crossover or case-crossover studies and studies using fixed effects analysis of repeated measures data or similar analytic methods- se $^{44}$ ) will be included. However, due to the possibility of carry-over effects or antidepressant withdrawal obscuring or reversing the observed association between antidepressant exposure and violence perpetration, sensitivity analysis will be used to examine the impact of including such studies. We will exclude open-label single-arm trials, case reports, case series, uncontrolled before/after studies, ecological studies and cross-sectional studies due to difficulties in 


\begin{tabular}{ll}
\hline Table 2 Antidepressants included in review \\
\hline Class & Generic drug name \\
$\begin{array}{ll}\text { Selective serotonin } & \text { Fluoxetine } \\
\text { reuptake inhibitors } & \text { Citalopram/escitalopram } \\
& \text { Fluvoxamine } \\
& \text { Sertraline } \\
& \text { Paroxetine } \\
& \text { Vortioxetine } \\
& \text { Vilazodone } \\
& \text { Venlafaxine } \\
& \text { Desvenlafaxine } \\
\text { Serotonin and } & \text { Duloxetine } \\
\text { norepinephrine reuptake } \\
\text { inhibitors }\end{array}$ \\
& Milnacipran/levomilnacipran
\end{tabular}

Tricyclic antidepressants Amitriptyline
Nortriptyline
Imipramine
Desipramine
Doxepin
Dothiepin
Protriptyline
Trimipramine
Clomipramine
Amoxapine
Lofepramine
Maprotiline
Tranylcypromine
Phenelzine
Selegiline
Isocarboxazid
Moclobemide
Anhibitors
Agomelatine
Bupropion
Nefazodone
Trazodone
Mirtazapine
Ketamine/esketamine
Mianserin
Brexanolone/allopregnanolone
Other

determining the direction of association. We will also exclude dissertations, conference abstracts and study registrations. We will not include previous systematic reviews, as not all included studies may meet out inclusion criteria. However, we will identify peer-reviewed studies related to any dissertations, conference abstracts or study registrations, and original studies referenced in related systematic reviews, and include any that met our inclusion criteria but were not identified by our search strategy.

\section{Search strategy and data management}

\section{Search strategy}

We will search key health and medical databases (MEDLINE, EMBASE, CINAHL, PsycINFO, PubMed and the Cochrane library) for peer-reviewed literature from inception of each database until most-recent available study on the date of conducting our final search. The initial search was performed on the 19 March 2020, and an update will be conducted on the 1 March 2021. We will use Cochrane-recommended search strategies to identify RCTs, ${ }^{45}$ and a version of Ovid's recommended observational study search filter, ${ }^{46}$ adapted for each database, to identify observational studies. The search strategy for MEDLINE (Ovid) is presented in table 3. The search strategy was developed in consultation with a librarian at the Murdoch Children's Research Institute. The reference and citation lists of all eligible studies will be screened to identify any additional relevant studies. Studies will be limited to those conducted on human participants. There will be no restrictions on the year or location of publication. We will only review English language abstracts. However, any full-text papers not in English that were identified via abstract search, grey literature search, reference list review or from author follow-up will be translated into English and reviewed.

To identify relevant trials that may have been conducted in the process of licencing or monitoring medications by national regulatory bodies, the following sources will be searched for grey literature: the FDA (https://www.fda. gov/home), the National Institute for Health and Care Excellence (NICE, https://www.nice.org.uk/), Pharmac (https://www.pharmac.govt.nz/), the PBAC (http:// www.pbs.gov.au/pbs/home), the Canadian Agency for Drugs and Technologies in Health (CADTH, https:// www.cadth.ca/) and Google Scholar.

\section{Study selection}

Identified studies will be imported into the citation management software Covidence (www.covidence.org) and duplicates will be removed. All titles and abstracts will be independently screened by the lead author (CK) and another member of the research team. After $15 \%$ of the papers identified through the search strategy have been double screened, we will reassess our inclusion and exclusion criteria to ensure they are relevant to the studies that have been identified. The reassessment will involve a discussion by two reviewers and an additional third author $(\mathrm{JY} / \mathrm{GN}-\mathrm{H} / \mathrm{JK})$ in which each study that was determined to be eligible for inclusion by at least one reviewer will be discussed, and any disagreements or uncertainty regarding the reasons for inclusion or exclusion will be resolved by consensus. Any changes or clarifications to the inclusion and exclusion criteria as a result of this discussion will be circulated to all members of the research team, and all team members will agree to these changes before screening continues. The updated inclusion and exclusion criteria will be used for the remaining title and abstract screening, which will be independently 


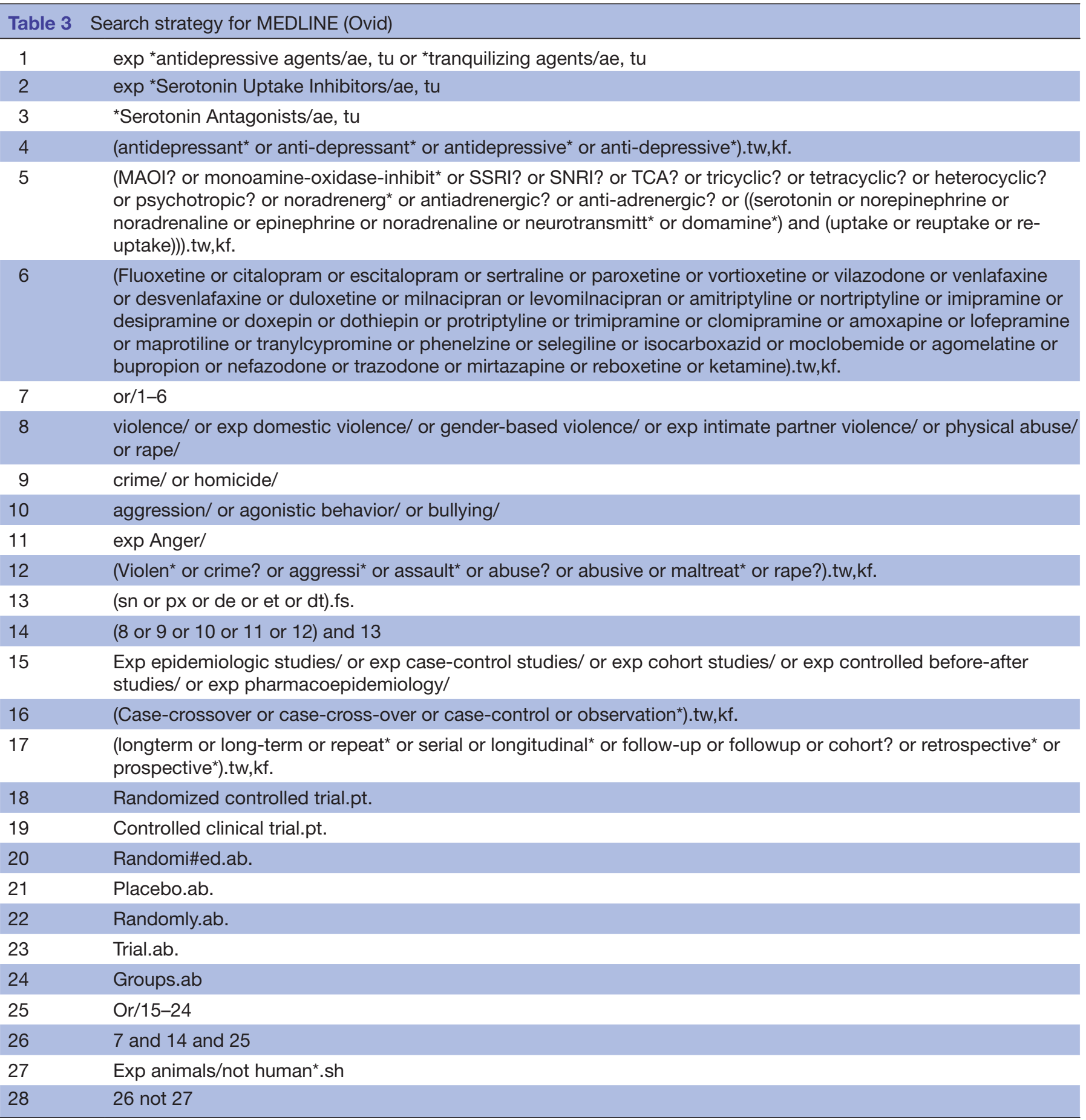

conducted by CK and one other member of the research team. Any uncertainty or disagreement over inclusion for the remaining studies will be resolved by a third author (JY/JK/GNH). The overall inter-rater reliability will be tested using Cohen's kappa statistic. ${ }^{47}$

Full-text articles will be screened by CK and one other member of research team, with a third reviewer resolving any conflicts. Where clarification is needed to determine eligibility, we will contact the original study authors. If multiple included studies use the same dataset (assessed using study titles, author names, locations and dates), the study using the longest duration of follow-up will be included (ie, the other study(ies) will be excluded.

\section{Data extraction}

Data will be extracted by one research team member using standardised, prespecified Excel forms developed by the research team. A second team member will check the extraction and amend any errors. A summary of the data extraction fields is presented in table 4 . We will contact study authors for additional information if the required data are missing, incomplete or unclear. 


\begin{tabular}{ll}
\hline Table 4 Extraction fields \\
\hline Domain & Extraction fields \\
Study details & Author(s) \\
& Year of publication \\
& Journal name \\
& Geographic location of study \\
& Year(s) of study \\
& Study type \\
& Population \\
Methods & Recruitment methods \\
& Type of antidepressant(s) \\
& Method(s) of measuring antidepressant use \\
& Type of comparator(s) \\
& Method(s) of measurement of comparator(s) \\
& Definition of violence \\
& Method of measuring violence \\
& Definition of aggression \\
& Method of measuring aggression \\
& Method of randomisation* \\
& Methods for avoiding confounding (covariate \\
adjustment, propensity score, etc.) & Blinding* \\
& Allocation procedure* \\
& Statistical analyses \\
& Number of partipants \\
&
\end{tabular}

$\begin{array}{ll}\text { Results } & \text { Number of participants } \\ \text { Age of participants (average and/or age } \\ \text { categories) } \\ \text { Proportion male and female } \\ \text { Median length of follow-up (and variance) } \\ \text { Attrition rates and/or exclusions after entry } \\ \text { Proportion missing data or loss to follow-up } \\ \text { Number exposed (total, by sex, by age) } \\ \text { Duration of antidepressant use and variance (total, } \\ \text { by sex, by age) } \\ \text { Number not exposed (total, by sex, by age) } \\ \text { Number with outcome (total, by sex, by age) } \\ \text { Number exposed with outcome (total, by sex, by } \\ \text { age) } \\ \text { Number not exposed with outcome (total, by sex, } \\ \text { by age) } \\ \text { Measure of association (and Cl, SE, p value) } \\ \text { Any treatment deviations, non-compliance or non- } \\ \text { adherence } \\ \text { Funding sources declared } \\ \text { interests }\end{array}$

${ }^{*}$ Relevant to randomised controlled trials.

†Relevant to observational studies.
Risk of bias assessment

The Methodological Standard for Epidemiological Research (MASTER) scale will be used to assess the quality of the included studies. ${ }^{48}$ The MASTER scale conceptualises quality scores as an indicator of a study's propensity towards bias, rather than an absolute measure of study biases. As such, it creates a means of ranking studies within a review based on their relative probability of bias. This ranking can be used to impute probability of bias variances for meta-analyses, without assuming the ranking is a direct measure of the absolute amount of bias present in any individual study. The MASTER scale uses a generic quality assessment tool across all study designs organised around seven methodological standards (equal recruitment, equal retention, equal ascertainment, equal implementation, equal prognosis, sufficient analysis and temporal precedence) that design-specific bias safeguards aim to fulfil.

The quality of studies and potential risks of bias will be discussed in text, and a quality summary score will be created based on the proportion of bias safeguards achieved in each study. As recommended by the Metaanalysis of Observational Studies in Epidemiology (MOOSE) study group, summary quality scores will not be used to weight analyses but will be used to guide a subgroup analysis of high-quality studies discussed below. ${ }^{49}$

\section{Statistical analysis}

We will provide a descriptive overview of characteristics of the included studies, including the quality of the studies, geographic coverage and types of measures used to assess exposure to antidepressants and aggression and violent outcomes.

$\mathrm{I}^{2}$ calculations will be used to assess heterogeneity. If there are sufficient studies that report measures of effect in a form which can be meta-analysed, a random metaanalysis will be used to estimate a pooled effect size. A random effects model is appropriate as it accounts for unexplained heterogeneity due to the diversity in study designs, treatment types and study populations which will be included in this review. ${ }^{50}$ To test for publication bias, visual inspection of a funnel plot will be performed if a sufficient number of studies is included ( $\mathrm{n} \sim 10$ or more). An Egger's test statistic will also be calculated.

In order to understand the impact of study design on these pooled effects measures, we will conduct a metaregression. ${ }^{51}$ If the number of included studies is sufficient, we will use meta-regression to investigate variation in effect according to age, sex, antidepressant type and subgroups of interest (including people with autism spectrum disorder, people with dementia, people with conduct disorder and people with a history of violent crime) and to investigate methodological effects including sample size, RCT versus observational design, length of follow-up and comparison condition. If there are differences by sex, antidepressant type or subgroups of interested, we will present analyses stratified by these factors. To examine 
the impact of study quality on outcomes, we will conduct a sensitivity analysis which includes only higher-quality papers (papers whose quality assessment score is in the top $30 \%$ of included papers).

If the included studies do not have sufficient data to warrant meta-analyses, results will be presented in a harvest plot, examining correlations between study characteristics (such as study design), quality and estimated measure of association ${ }^{52}$ and will be complemented by a narrative description of the included study findings.

\section{Ethics and dissemination}

To our knowledge, this review will be the first to systematically synthesise research from RCTs and observational studies on the association between antidepressants and violence perpetration. The findings of this review may be useful to academic researchers, medication prescribers, drug regulatory bodies, and people who have been prescribed antidepressants. We will disseminate our findings by publishing results in a peer-reviewed journal and presenting results at conferences. We will provide our results to relevant professional organisations, stakeholders, and policymakers for dissemination to their members, and publish a plain language summary that links to the main publication.

As this is a review of studies which have already been subject to ethics approval, this study does not require ethics approval.

\section{Author affiliations}

1Justice Health Unit, Centre for Health Equity, Melbourne School of Population and Global Health, The University of Melbourne, Melbourne, Victoria, Australia ${ }^{2}$ Department of Psychological Medicine, University of Otago, Christchurch, New Zealand

${ }^{3}$ Centre for Adolescent Health, Murdoch Childrens Research Institute, Parkville, Victoria, Australia

${ }^{4}$ Department of Psychological Medicine, University of Otago, Wellington, New Zealand

${ }^{5}$ Centre for Health Policy, Melbourne School of Population and Global Health, The University of Melbourne, Melbourne, Victoria, Australia

${ }^{6}$ Psychiatry, University of Oxford, Oxford, UK

${ }^{7}$ Melbourne School of Psychological Sciences, The University of Melbourne, Melbourne, Victoria, Australia

${ }^{8}$ Health Service and Population Research Department, Institute of Psychiatry, Psychology and Neuroscience, King's College London, London, UK

${ }^{9}$ Mater Research Institute-UQ, University of Queensland, Brisbane, Queensland, Australia

${ }^{10}$ Griffith Criminology Institute, Griffith University, Brisbane, Queensland, Australia

${ }^{11}$ School of Public Health and Preventative Medicine, Monash University, Melbourne, Victoria, Australia

${ }^{12}$ School of Population and Global Health, The University of Western Australia, Perth, Western Australia, Australia

${ }^{13}$ National Drug Research Institute, Curtin University, Perth, Western Australia, Australia

\section{Twitter Stuart A Kinner @KinnerStuart}

Contributors CK and JY developed the original research proposal. CK, JY, JAF, GN-H, MW, SF and JK contributed to the design of the project. CK and MW developed the search strategy with input from JY, JAF, GN-H, MW and JK. CK wrote the initial draft manuscript and JY, JAF, GN-H, MW, SF, RB, JK and SAK contributed significantly to drafting and editing the manuscript. All authors approved the final manuscript.
Funding JY receives salary and research support from a National Health and Medical Research Council Investigator Grant (NTG1178027). SAK receives salary and research support from a National Health and Medical Research Council Senior Research Fellowship (GNT1078168). Apart from the funding sources acknowledged above, this research received no specific grant from any funding agency in the public, commercial or not-for-profit sectors.

Competing interests None declared.

Patient and public involvement Patients and/or the public were not involved in the design, or conduct, or reporting, or dissemination plans of this research.

Patient consent for publication Not required.

Provenance and peer review Not commissioned; externally peer reviewed.

Open access This is an open access article distributed in accordance with the Creative Commons Attribution Non Commercial (CC BY-NC 4.0) license, which permits others to distribute, remix, adapt, build upon this work non-commercially, and license their derivative works on different terms, provided the original work is properly cited, appropriate credit is given, any changes made indicated, and the use is non-commercial. See: http://creativecommons.org/licenses/by-nc/4.0/.

\section{ORCID iDs}

Claire Keen http://orcid.org/0000-0002-4875-1300

Melissa Willoughby http://orcid.org/0000-0002-4360-2605

Josh Knight http://orcid.org/0000-0001-5268-8263

Jesse T Young http://orcid.org/0000-0001-5702-372X

\section{REFERENCES}

1 Moore M, Yuen HM, Dunn N, et al. Explaining the rise in antidepressant prescribing: a descriptive study using the general practice research database. BMJ 2009;339:b3999-b.

2 Hemels MEH, Koren G, Einarson TR. Increased use of antidepressants in Canada: 1981-2000. Ann Pharmacother 2002;36:1375-9.

3 Pratt L, Brody D, Gu Q. Antidepressant use among persons aged 12 and over: United States, 2011-2014. In: No.283 NDB. Maryland: Centers for Disease Control and Prevention, 2017.

4 OECD. Health at a glance 2019: OECD indicators. Paris: OECD Publishing, 2019.

5 lacobucci G. NHS prescribed record number of antidepressants last year. BMJ 2019;364:11508.

6 Australian Institute of Health and Welfare. Mental health services in Australia Canberra: AlHW, 2019. Available: https://www.aihw.gov.au/ reports/mental-health-services/mental-health-services-in-australia/ report-contents/mental-health-related-prescriptions/prescriptions

7 Wong J, Motulsky A, Eguale T, et al. Treatment indications for antidepressants prescribed in primary care in Quebec, Canada, 2006-2015. JAMA 2016;315:2230-2.

8 Dahlberg LL, Krug EG. Violence a global public health problem. Ciência \& Saúde Coletiva 2006;11:277-92.

9 Haagsma JA, Graetz N, Bolliger I, et al. The global burden of injury: incidence, mortality, disability-adjusted life years and time trends from the global burden of disease study 2013. Inj Prev 2016;22:3-18.

10 James SL, Abate D, Abate $\mathrm{KH}$, et al. Global, regional, and national incidence, prevalence, and years lived with disability for 354 diseases and injuries for 195 countries and territories, 1990-2017: a systematic analysis for the global burden of disease study 2017 . Lancet 2018;392:1789-858.

11 Sheard MH, Marini JL, Bridges Cl, et al. The effect of lithium on impulsive aggressive behavior in man. Am J Psychiatry 1976;133:1409-13.

12 Eichelman B. Toward a rational pharmacotherapy for aggressive and violent behavior. Psychiatric Services 1988;39:31-9.

13 Kraus JE, Sheitman BB. Clozapine reduces violent behavior in heterogeneous diagnostic groups. J Neuropsychiatry Clin Neurosci 2005;17:36-44.

14 Krakowski MI, Czobor P, Citrome L, et al. Atypical antipsychotic agents in the treatment of violent patients with schizophrenia and schizoaffective disorder. Arch Gen Psychiatry 2006;63:622-9.

15 Stanford MS, Anderson NE, Lake SL, et al. Pharmacologic treatment of impulsive aggression with antiepileptic drugs. Curr Treat Options Neurol 2009;11:383-90.

16 Goedhard LE, Stolker JJ, Heerdink ER, et al. Pharmacotherapy for the treatment of aggressive behavior in general adult psychiatry: a systematic review. J Clin Psychiatry 2006;67:1013-24.

17 Schofield PW, Xu A, Simpson P, et al. Pharmacotherapy to reduce violent offending? Offenders might be interested 2019;53:697-8. 
18 Butler T, Schofield PW, Greenberg D, et al. Reducing impulsivity in repeat violent offenders: an open label trial of a selective serotonin reuptake inhibitor. Aust N Z J Psychiatry 2010;44:1137-43.

19 Walsh MT, Dinan TG. Selective serotonin reuptake inhibitors and violence: a review of the available evidence. Acta Psychiatr Scand 2001;104:84-91.

20 Bond AJ. Antidepressant treatments and human aggression. Eur J Pharmacol 2005;526:218-25.

21 Carrillo M, Ricci LA, Coppersmith GA, et al. The effect of increased serotonergic neurotransmission on aggression: a critical metaanalytical review of preclinical studies. Psychopharmacology 2009;205:349-68.

22 Haden SC, Scarpa A. The noradrenergic system and its involvement in aggressive behaviors. Aggression and Violent Behavior 2007;12:1-15.

23 Reist C, Nakamura K, Sagart E. Impulsive aggressive behavior: openlabel treatment with citalopram 2002;81.

24 Cherek DR, Lane SD, Pietras CJ, et al. Effects of chronic paroxetine administration on measures of aggressive and impulsive responses of adult males with a history of conduct disorder. Psychopharmacology 2002;159:266-74.

25 Coccaro EF, Lee RJ, Kavoussi RJ. A double-blind, randomized, placebo-controlled trial of fluoxetine in patients with intermittent explosive disorder. J Clin Psychiatry 2009;70:653-62.

26 Romero-Martínez Ángel, Murciano-Martí S, Moya-Albiol L. Is sertraline a good pharmacological strategy to control anger? results of a systematic review. Behav Sci 2019;9:57.

27 Sharma T, Guski LS, Freund N, et al. Suicidality and aggression during antidepressant treatment: systematic review and metaanalyses based on clinical study reports 2016;352:i65.

28 Bielefeldt Andreas $\varnothing$, Danborg PB, Gøtzsche PC. Precursors to suicidality and violence on antidepressants: systematic review of trials in adult healthy volunteers. J R Soc Med 2016;109:381-92.

29 Molero Y, Lichtenstein P, Zetterqvist J, et al. Selective serotonin reuptake inhibitors and violent crime: a cohort study. PLoS Med 2015;12:e1001875

30 Healy D, Herxheimer A, Menkes DB. Antidepressants and violence: problems at the interface of medicine and law. PLoS Med 2006;3:e372.

31 Suicidality BPR. Violence and mania caused by selective serotonin reuptake inhibitors (SSRIs): a review and analysis. International Journal of Risk \& Safety in Medicine 2003;16:31-49.

32 Hieronymus F, Lisinski A, Näslund J, et al. Multiple possible inaccuracies cast doubt on a recent report suggesting selective serotonin reuptake inhibitors to be toxic and ineffective. Acta Neuropsychiatr 2018;30:244-50.

33 Katakam KK, Sethi NJ, Jakobsen JC, et al. Great boast, small roast on effects of selective serotonin reuptake inhibitors: response to a critique of our systematic review. Acta Neuropsychiatr 2018;30:251-65.

34 Stone M. Suicidality and aggression during antidepressant treatment authors misinterpreted earlier paper from the FDA. BMJ 2016;i:906.
35 Dubicka B, Cole-King A, Reynolds S, et al. Paper on suicidality and aggression during antidepressant treatment was flawed and the press release was misleading. BMJ 2016;352:911.

36 Goodwin GM, Nutt D. Antidepressants; what's the beef? Acta Neuropsychiatr 2019;31:59-60.

37 Barbui C, Esposito E, Cipriani A. Selective serotonin reuptake inhibitors and risk of suicide: a systematic review of observational studies. CMAJ 2009;180:291-7.

38 Danborg PB, Valdersdorf M, Gøtzsche PC. Long-Term harms from previous use of selective serotonin reuptake inhibitors: a systematic review. Int J Risk Saf Med 2019;30:59-71.

39 Foulds J, Young JT, et al, Pharmacotherapy for incarcerated people with a history of violence: Response to commentary by Schofield. Aust N Z J psychiatry 2019:4867419885175.

40 Moher D, Shamseer L, Clarke M, et al. Preferred reporting items for systematic review and meta-analysis protocols (PRISMA-P) 2015 statement. Syst Rev 2015;4:1.

41 Rutherford A, Zwi AB, Grove NJ, et al. Violence: a glossary. J Epidemiol Community Health 2007;61:676-80.

42 World Health Organization. World report on violence and health. Geneva: WHO, 2002

43 Ryan R, Hill S, Broclain D, et al. Study design guide. Cochrane consumers and communication review group, 2013.

44 Gunasekara FI, Richardson K, Carter K, et al. Fixed effects analysis of repeated measures data. Int J Epidemiol 2014;43:264-9.

45 Cochrane Handbook for Systematic Reviews of Interventions Version 6.0 (updated July 2019) 2019.

46 Ovid Expert Searches - Health Science [Internet], 2019. Available: http://resourcecenter.ovid.com/site/resources/expert search/ healthexp.html\#OvidFilters

47 McHugh ML. Interrater reliability: the kappa statistic. Biochem Med 2012;22:276-82.

48 Stone JC, Glass K, Clark J, et al. A unified framework for bias assessment in clinical research. Int J Evid Based Healthc 2019;17:106-120.

49 Stroup DF, Berlin JA, Morton SC, et al. Meta-analysis of observational studies in epidemiology: a proposal for reporting. Meta-analysis of Observational Studies in Epidemiology (MOOSE) group. JAMA 2000;283:2008-12.

50 Barili F, Parolari A, Kappetein PA, et al. Statistical primer: heterogeneity, random- or fixed-effects model analyses? Interact Cardiovasc Thorac Surg 2018;27:317-21.

51 Baker WL, Michael White C, Cappelleri JC. From the health outcomes $\mathrm{P}$, et al. understanding heterogeneity in meta-analysis: the role of meta-regression. International Journal of Clinical Practice 2009;63:1426-34.

52 Ogilvie D, Fayter D, Petticrew M, et al. The harvest plot: a method for synthesising evidence about the differential effects of interventions. BMC Med Res Methodol 2008;8:8. 